\title{
A Magnus- and Fer-Type Formula in Dendriform Algebras
}

\author{
Kurusch Ebrahimi-Fard • Dominique Manchon
}

Received: 17 July 2007 / Revised: 10 December 2007 / Accepted: 20 December 2007 /

Published online: 6 February 2008

(C) SFoCM 2008

\begin{abstract}
We provide a refined approach to the classical Magnus (Commun. Pure Appl. Math. 7:649-673, 1954) and Fer expansion (Bull. Classe Sci. Acad. R. Belg. $44: 818-829,1958)$, unveiling a new structure by using the language of dendriform and pre-Lie algebras. The recursive formula for the logarithm of the solutions of the equations $X=1+\lambda a \prec X$ and $Y=1-\lambda Y \succ a$ in $A[[\lambda]]$ is provided, where $(A, \prec, \succ)$ is a dendriform algebra. Then we present the solutions to these equations as an infinite product expansion of exponentials. Both formulae involve the pre-Lie product naturally associated with the dendriform structure. Several applications are presented.
\end{abstract}

Keywords Linear differential equation · Linear integral equation · Magnus expansion · Fer expansion - Dendriform algebra $\cdot$ Pre-Lie algebra $\cdot$ Rota-Baxter algebra $\cdot$ Binary rooted trees

Mathematics Subject Classification (2000) Primary: 16W25 - 17A30 - 17D25 37C10 - Secondary: 05C05 $\cdot 81 \mathrm{~T} 15$

Communicated by Arieh Iserles.

K. Ebrahimi-Fard ( $\varangle)$

Max-Planck-Institut für Mathematik, Vivatsgasse 7, 53111 Bonn Germany e-mail: kurusch@mpim-bonn.mpg.de

D. Manchon

Université Blaise Pascal, C.N.R.S.-UMR 6620, 63177 Aubière, France

e-mail: manchon@math.univ-bpclermont.fr 


\section{Introduction}

Let us start by emphasizing that the results presented in the sections following this Introduction are an extension of findings obtained by the authors together with Patras in an earlier work [8]. The underlying theme of our paper is to bring together Magnus', Fer's and Baxter's classical work on linear differential equations, respectively, the corresponding integral equations, using the language of Loday's dendriform algebras. Skipping details, in this Introduction, we try to sketch briefly the general picture behind our results.

Recall that Magnus [20] and Fer [9], as well as Baxter [2], start their papers by recalling the classical initial value problem

$$
\dot{\Phi}(t):=\frac{d}{d t} \Phi(t)=\Psi(t) \Phi(t), \quad \Phi(0)=1
$$

Magnus considers it in a noncommutative context, i.e., $A:=\Psi$ and $Y:=\Phi$ are supposed to be linear operators depending on a real variable $t$. Here, 1 denotes the identity operator. For the linear operator $\Omega(t)$, depending on $A$ and with $\Omega(0)=0$, such that

$$
Y(t)=\exp \left(\int_{0}^{t} \dot{\Omega}(s) d s\right)=\sum_{n \geq 0} \frac{\Omega(t)^{n}}{n !},
$$

Magnus obtains a differential equation leading to the recursively defined expansion named after him:

$$
\Omega(t)=\int_{0}^{t} \dot{\Omega}(s) d s=\int_{0}^{t} A(s) d s+\int_{0}^{t} \sum_{n>0} \frac{B_{n}}{n !} a d_{\int_{0}^{s} \dot{\Omega}(u) d u}^{(n)}[A(s)] d s .
$$

Here, as usual, $a d_{f}[g]:=f g-g f:=[f, g]$. The coefficients $B_{n}$ are the Bernoulli numbers defined via the generating series

$$
\frac{z}{\exp (z)-1}=\sum_{m \geq 0} \frac{B_{m}}{m !} z^{m}=1-\frac{1}{2} z+\frac{1}{12} z^{2}-\frac{1}{720} z^{4}+\cdots .
$$

Observe that $B_{2 m+3}=0, m \geq 0$ and recall for later use

$$
\frac{\exp (z)-1}{z}=\int_{0}^{1} \exp (s z) d s
$$

For more details, see for instance [10, 12, 14, 16, 20, 21, 23, 27, 28]. Observe also that Magnus' expansion (2) reduces to $\int_{0}^{t} A(s) d s$ if all commutators disappear, e.g., in a commutative setting, leading to the classical exponential solution

$$
Y(t)=\exp \left(\int_{0}^{t} A(s) d s\right)
$$


for the initial value problem (1). Indeed, the reason for this exponential solution is simply encoded in the integration by parts rule for $I(A)(t):=\int_{0}^{t} A(s) d s$ :

$$
(I(A)(t))^{n}=n ! \underbrace{I(A I(A \cdots I(A) \cdots))}_{n \text {-times }}(t) .
$$

Fer's [9] approach to solve the classical initial value problem (1), which was rediscovered by Iserles [11] and further explored by Zanna [29], and Munthe-Kaas and Zanna [22], see [13] for more details, is somewhat different. His ansatz is,

$$
Y=\exp \left(\int_{0}^{t} A(s) d s\right) V(t)
$$

which leads to the following differential equation for the "correction" operator $V(t)$ :

$$
\dot{V}(t)=\left(\sum_{k>0} \frac{(-1)^{k} k}{(k+1) !} a d_{\int_{0}^{t} A(s) d s}^{(k)}[A(t)]\right) V(t), \quad V(0)=1 .
$$

Hence, an iteration of this method leads to the Fer expansion

$$
\begin{aligned}
Y(t)= & \exp \left(\int_{0}^{t} U_{0}^{\prime}(s) d s\right) \exp \left(\int_{0}^{t} U_{1}^{\prime}(s) d s\right) \\
& \times \exp \left(\int_{0}^{t} U_{2}^{\prime}(s) d s\right) \cdots \exp \left(\int_{0}^{t} U_{n}^{\prime}(s) d s\right) \cdots,
\end{aligned}
$$

where we use Fer's recursion

$$
U_{m+1}^{\prime}(t):=\left(\sum_{k>0} \frac{(-1)^{k} k}{(k+1) !} a d_{\int_{0}^{t} U_{m}^{\prime}(s) d s}^{(k)}\left[U_{m}^{\prime}(t)\right]\right), \quad U_{0}^{\prime}(t):=A(t) .
$$

Taking an algebra-combinatorial perspective on these methods, we should underline at this point that we completely skip the analytical and numerical aspects of these expansions, which are beyond doubt of crucial importance in applications. For this purpose and related aspects, we refer the interested reader to the aforementioned references, e.g., see [3, 14, 23]. However, at the end of this work, we report on an observation which may be of interest in this context and which we plan to further explore in the near future.

Baxter [2] considers (1) in a commutative setting, i.e., for continuous scalar functions $a:=\Psi$ and $y:=\Phi$ depending on $t$. However, his starting point is the corresponding integral equation

$$
y(t)=1+\int_{0}^{t} a(s) y(s) d s,
$$

and its exponential solution. Slightly deviating from Baxter's original approach, we generalize (6) to a formal power series ring, $W[[\lambda]]$, where $W$ is a commutative unital algebra over a field $k$ with a $k$-linear map $R: W \rightarrow W$ replacing the integral map

$$
Y=1+\lambda R(a Y),
$$


$a \in W$ fixed. Here, 1 is the unit in the algebra $W$. The map $R$ is supposed to satisfy the relation

$$
R(x) R(y)=R(R(x) y+x R(y)+\theta x y),
$$

where the parameter $\theta$ is a fixed scalar in $k$, called the weight of $R$. One may think of (8) as a generalized integration by parts identity. Indeed, the reader will have no difficulty in checking duality of (8) with the "skewderivation" rule

$$
\partial(f g)=\partial(f) g+f \partial(g)+\theta \partial(f) \partial(g) .
$$

For example, the finite difference operator of step $-\theta$, given by $\partial f(x):=\theta^{-1} \times$ $(f(x-\theta)-f(x))$, is a skewderivation. On a suitable class of functions, the summation operator

$$
S(f)(x):=\sum_{n \geq 1} \theta f(x+\theta n) .
$$

satisfies relation (8). Moreover

$$
\begin{aligned}
S \partial(f)(x) & =\sum_{n \geq 1} \theta \partial(f)(x+\theta n) \\
& =\sum_{n \geq 1} \theta \frac{f(x+\theta n-\theta)-f(x+\theta n)}{\theta} \\
& =\sum_{n \geq 1} f(x+\theta(n-1))-f(x+\theta n) \\
& =\sum_{n \geq 0} f(x+\theta n)-\sum_{n \geq 1} f(x+\theta n)=f(x) .
\end{aligned}
$$

And as the operator $\partial$ is linear, we find as well $\partial S(f)=f$. Obviously, the skewderivation rule reduces to Leibniz' rule for $\theta=0$, e.g., see [4] for more details.

The exponential solution of equation (7) in $W[[\lambda]]$ can be seen as a natural generalization of the classical exponential solution (3) taking into account the weighted term in identity (8):

$$
Y=1+\sum_{n>0} \lambda^{n} \underbrace{R(a R(a \cdots R(a)}_{n \text {-times }}) \cdots)=\exp \left(R\left(\frac{\log (1+\theta a \lambda)}{\theta}\right)\right) .
$$

The second equality is generally known as Spitzer's classical identity, see $[1,2,7,25]$. In fact, expanding the logarithm and the exponential on the right-hand side, it follows, by comparing order by order in the parameter $\lambda$, the infinite set of identities in $W[[\lambda]]$.

Remember that the Riemann integral map $I:=\int_{0}^{t}$ satisfies identity (8) for the weight $\theta=0$ (integration by parts). In this particular case, observe that

$$
\theta^{-1} \log (1+\theta a \lambda)=-\sum_{n>0} \frac{(-\theta)^{n-1}}{n}(a \lambda)^{n} \stackrel{\theta \downarrow 0}{\longrightarrow} a \lambda .
$$


Hence, the exponential on the right-hand side of identity (10) reduces to the classical exponential solution (3).

Having Magnus' work in mind, it seems natural to ask for a noncommutative version of Spitzer's classical identity. In fact, (10) is only true when the underlying algebra is commutative, i.e., when (8) implies

$$
R(a)^{2}=2 R(a R(a))+\theta R\left(a^{2}\right) .
$$

Its generalization to arbitrary weight $\theta$ Rota-Baxter algebras, i.e., noncommutative algebras with a map $R$ satisfying relation (8) can be found in [7]. Similarly to Magnus' expansion (2), it relies on a particular nonlinear recursion $\chi_{\theta}$, which we call weight $\theta$ BCH-recursion as it is based on the Baker-Campbell-Hausdorff formula

$$
\chi_{\theta}(a)=a+\frac{1}{\theta} \operatorname{BCH}\left(\theta a, R \circ \chi_{\theta}(a)\right) .
$$

We then find the noncommutative Spitzer identity

$$
Y=1+\sum_{n>0} \lambda^{n} \underbrace{R(a R(a \cdots R(a)}_{n \text {-times }}) \cdots)=\exp \left(R\left(\chi_{\theta}\left(\frac{\log (1+\theta a \lambda)}{\theta}\right)\right)\right) .
$$

We refer the reader to Sect. 5 for more details. One may also consult $[4,7]$.

At first sight, the $\theta \mathrm{BCH}$ - and Magnus recursion, (11) respectively (2), look different, but it is the goal of this work to show how they are related. Indeed, in [7] it was already shown that (11) reduces to Magnus' formula (2) in the corresponding limit $\theta \rightarrow 0$. Here, on the contrary, we will show how to get (11) from the limit case $\theta=0$. After this has been achieved, we provide a refined picture of Fer's expansion (5) in purely algebraic terms leading to an infinite product expansion of the solution to (7). These results are achieved in the context of the generalized integration by parts rule (8). We emphasize the use of Loday's dendriform algebras [18] which appears to be well suited.

Recall that a dendriform algebra is an associative algebra with two nonassociative operations, written $\prec$ and $\succ$ satisfying three rules. The two products add to form the product of the algebra. At the same time, they define a left and right pre-Lie product on the same algebra. Loday recently introduced this notion in connection with dialgebra structures $[18,19]$. The commutative version of dendriform algebra is called "dual Leibniz algebra" or "Zinbiel algebra" by Loday [17] (see also [26]). Temporarily, this algebra, respectively, its product had been called "chronological algebra," respectively, "chronological product" in the context of control theory, e.g., see [15] for more details. The key point from our perspective is the intimate relation between associative algebras, equipped with a map satisfying relation (8) and such dendriform algebras. We will see that this connection renders dendriform algebras a suitable setting to encode algebraic structures related to the integral equations corresponding to linear differential equations. At the end, we will indicate that this approach unveils a hitherto hidden structure in Fer's and Magnus' original work leading to a reduction in number of commutator terms. The reader may also look into [4] for related issues.

This work is organized as follows. In Sect. 2, we recall the notion of dendriform algebra and introduce two particular dendriform power sums expansions. Section 3 
contains the first main result of this work, that is, we present the central object, a new pre-Lie Magnus type recursion, and show that its exponentiation solves the aforementioned pair of dendriform power sums expansions. Then in Sect. 4, we introduce the pre-Lie Fer recursion as the second main result, leading to an infinite product expansion of exponentials for these solutions. Finally, in Sect. 5, we provide some applications. We finish the article with an observation indicating hitherto overlooked new structural properties of the classical Magnus and Fer expansions due to the extra pre-Lie relation, which leads to a reduction in the number of terms in these expansions.

\section{Dendriform Power Sums Expansions}

Let $k$ be a field of characteristic zero. Recall that a dendriform algebra [18] over $k$ is a $k$-vector space $A$ endowed with two bilinear operations $\prec$ and $\succ$ subject to the three axioms below:

$$
\begin{aligned}
& (a \prec b) \prec c=a \prec(b * c), \\
& (a \succ b) \prec c=a \succ(b \prec c), \\
& a \succ(b \succ c)=(a * b) \succ c .
\end{aligned}
$$

In the commutative case, the left and right operations are further required to be identified, so that $x \succ y=y \prec x$. One can show that these relations yield associativity for the product

$$
a * b:=a \prec b+a \succ b .
$$

Example 1 As a guiding example we regard an algebra $F$ of operator-valued functions on the real line, closed under integrals $\int_{0}^{x}$, say, smooth $n \times n$ matrix-valued functions. Then, $D_{F}=(F, \prec, \succ)$ is a dendriform algebra for the operations

$$
(A \prec B)(x):=A(x) \cdot \int_{0}^{x} B(y) d y, \quad(A \succ B)(x):=\int_{0}^{x} A(y) d y \cdot B(x)
$$

with $A, B \in F$. One verifies the dendriform axioms using the integration by parts rule. For instance, in this setting, the dendriform relation (13) means

$$
\begin{aligned}
& \left(A(x) \cdot \int_{0}^{x} B(u) d u\right) \cdot \int_{0}^{x} C(v) d v \\
& =A(x) \cdot \int_{0}^{x}\left(B(u) \cdot \int_{0}^{u} C(v) d v+\int_{0}^{u} B(v) d v \cdot C(u)\right) d u
\end{aligned}
$$

for $A, B, C \in F$. The associative product in this dendriform algebra is then written

$$
(A * B)(x):=A(x) \cdot \int_{0}^{x} B(y) d y+\int_{0}^{x} A(y) d y \cdot B(x) .
$$


Let us remark that a commutative algebra $\left(F, \int_{0}^{x}\right)$ naturally provides a commutative dendriform algebra.

Let us return to the dendriform axioms. One shows that at the same time the dendriform relations imply that the bilinear operations $\triangleright$ and $\triangleleft$ defined by

$$
a \triangleright b:=a \succ b-b \prec a, \quad a \triangleleft b:=a \prec b-b \succ a
$$

are left pre-Lie and right pre-Lie, respectively, which means that we have

$$
\begin{aligned}
& (a \triangleright b) \triangleright c-a \triangleright(b \triangleright c)=(b \triangleright a) \triangleright c-b \triangleright(a \triangleright c), \\
& (a \triangleleft b) \triangleleft c-a \triangleleft(b \triangleleft c)=(a \triangleleft c) \triangleleft b-a \triangleleft(c \triangleleft b) .
\end{aligned}
$$

In the setting of our guiding Example 1, these pre-Lie products are

$$
\begin{aligned}
& (A \triangleleft B)(x):=A(x) \cdot \int_{0}^{x} B(y) d y-\int_{0}^{x} B(y) d y \cdot A(x), \\
& (A \triangleright B)(x):=\int_{0}^{x} A(y) d y \cdot B(x)-B(x) \cdot \int_{0}^{x} A(y) d y .
\end{aligned}
$$

The associative operation $*$ and the pre-Lie operations $\triangleright, \triangleleft$ all define the same Lie bracket:

$$
[a, b]:=a * b-b * a=a \triangleright b-b \triangleright a=a \triangleleft b-b \triangleleft a .
$$

Loday and Ronco introduced in [19] the notion of tridendriform algebra $T$ equipped with three operations, $<,>$ and $\bullet$, satisfying seven dendriform type axioms:

$$
\begin{array}{ll}
(x<y)<z=x<(y \star z), & (x>y)<z=x>(y<z), \\
(x \star y)>z=x>(y>z), & (x>y) \bullet z=x>(y \bullet z), \\
(x<y) \bullet z=x \bullet(y>z), & (x \bullet y)<z=x \bullet(y<z), \\
(x \bullet y) \bullet z=x \bullet(y \bullet z), &
\end{array}
$$

yielding an associative product $x \star y:=x<y+x>y+x \bullet y$. First, observe that the category of dendriform algebras can be identified with the subcategory of objects in the category of tridendriform algebras with $\bullet=0$. Moreover, one readily verifies for a tridendriform algebra $(T,<,>, \bullet)$ that $\left(D_{T}, \prec_{\bullet}, \succ\right)$, where $\prec_{\bullet}:=<+\bullet$ and $\succ:=>$, is a dendriform algebra [6].

Example 2 The summation operation (9) for $\theta=1$ on a suitable algebra $F$ of functions provides a natural example for such a tridendriform algebra $T_{F}=(F,<,>, \bullet)$ :

$$
\begin{aligned}
& (A<B)(x):=A(x) \cdot S(B)(x), \quad(A>B)(x):=S(A)(x) \cdot B(x), \\
& (A \bullet B)(x):=A(x) \cdot B(x) .
\end{aligned}
$$


One verifies without difficulty the tridendriform relations. For instance, the first relation simply encodes

$$
(A(x) \cdot S(B)(x)) \cdot S(C)(x)=A(x) \cdot(S(B \cdot S(C))(x)+S(S(B) \cdot C)(x)+S(B \cdot C)(x))
$$

which is identity (8). The associative product in this tridendriform algebra then becomes

$$
(A \star B)(x)=A(x) \cdot S(B)(x)+S(A)(x) \cdot B(x)+A(x) \cdot B(x) .
$$

Within this example, the reader may want to convince him or herself that $D_{T_{F}}=$ $\left(F, \prec_{\bullet}, \succ\right)$, where $\prec_{\bullet}:=<+\bullet$, i.e.

$$
(A \prec \bullet B)(x)=(A<B)(x)+(A \bullet B)(x)
$$

and $\succ:=>$, is a dendriform algebra.

One anticipates that dendriform algebras provide an elegant setting for a refined encoding of fundamental structures underlying integration and summation operations. In fact, further below we will show that this is true for a much larger class of operators, i.e., those characterized by identity (8).

Let $\bar{A}=A \oplus k . \mathbf{1}$ be our dendriform algebra augmented by a unit $\mathbf{1}$ :

$$
a \prec \mathbf{1}:=a=: \mathbf{1} \succ a \quad \mathbf{1} \prec a:=0=: a \succ \mathbf{1},
$$

implying $a * \mathbf{1}=\mathbf{1} * a=a$. Note that $\mathbf{1} * \mathbf{1}=\mathbf{1}$, but that $\mathbf{1} \prec \mathbf{1}$ and $\mathbf{1} \succ \mathbf{1}$ are not defined $[5,24]$. We recursively define the following set of elements of $\bar{A}[[\lambda]]$ for a fixed $a \in A$ :

$$
\begin{aligned}
& w_{\prec}^{(0)}(a):=\mathbf{1}=: w_{\succ}^{(0)}(a), \quad w_{\prec}^{(n)}(a):=a \prec\left(w_{\prec}^{(n-1)}(a)\right), \\
& w_{\succ}^{(n)}(a):=\left(w_{\succ}^{(n-1)}(a)\right) \succ a .
\end{aligned}
$$

Let us define the exponential and logarithm map in terms of the associative product (16), $\exp ^{*}(x):=\sum_{n \geq 0} x^{* n} / n !, \log ^{*}(1+x):=-\sum_{n>0}(-1)^{n} x^{* n} / n$, respectively. In the following we first give a recursive expression for the logarithm of the solutions of the following two equations for a fixed $a \in A$ :

$$
X=\mathbf{1}+\lambda a \prec X, \quad Y=\mathbf{1}-Y \succ \lambda a
$$

in $\bar{A}[[\lambda]]$, in terms of the left pre-Lie product $\triangleright$. This will in particular encompass the Magnus expansion for the logarithm of a solution of a linear first-order homogeneous differential equation in a noncommutative algebra [20]. Then we present the solutions of the two equations as an infinite product expansion of the exponential, which encompasses Fer's solution to a linear first-order homogeneous differential equation [9].

Remark 1 For later use, we may also write

$$
(X-\mathbf{1})=\lambda a+\lambda a \prec(X-\mathbf{1}) \quad \text { resp. } \quad(Y-\mathbf{1})=-\lambda a-(Y-\mathbf{1}) \succ \lambda a .
$$




\section{The Pre-Lie Magnus Expansion}

Formal solutions to (23) are given by

$$
X=\sum_{n \geq 0} \lambda^{n} w_{\prec}^{(n)}(a) \quad \text { resp. } \quad Y=\sum_{n \geq 0}(-\lambda)^{n} w_{\succ}^{(n)}(a) .
$$

Let us introduce the following operators in $(A, \prec, \succ)$, where $a$ is any element of $A$ :

$$
\begin{aligned}
& L_{\prec}[a](b):=a \prec b, \quad L_{\succ}[a](b):=a \succ b, \\
& R_{\prec}[a](b):=b \prec a, \quad R_{\succ}[a](b):=b \succ a, \\
& L_{\triangleleft}[a](b):=a \triangleleft b, \quad L_{\triangleright}[a](b):=a \triangleright b, \\
& R_{\triangleleft}[a](b):=b \triangleleft a, \quad R_{\triangleright}[a](b):=b \triangleright a .
\end{aligned}
$$

Theorem 2 Let $\Omega^{\prime}:=\Omega^{\prime}(\lambda a), a \in A$, be the element of $\lambda \bar{A}[[\lambda]]$ such that $X=$ $\exp ^{*}\left(\Omega^{\prime}\right)$ and $Y=\exp ^{*}\left(-\Omega^{\prime}\right)$, where $X$ and $Y$ are the solutions of the two equations (23), respectively. This element obeys the following recursive equation:

$$
\Omega^{\prime}(\lambda a)=\frac{R_{\triangleleft}\left[\Omega^{\prime}\right]}{1-\exp \left(-R_{\triangleleft}\left[\Omega^{\prime}\right]\right)}(\lambda a)=\sum_{m \geq 0}(-1)^{m} \frac{B_{m}}{m !} R_{\triangleleft}\left[\Omega^{\prime}\right]^{m}(\lambda a),
$$

or alternatively

$$
\Omega^{\prime}(\lambda a)=\frac{L_{\triangleright}\left[\Omega^{\prime}\right]}{\exp \left(L_{\triangleright}\left[\Omega^{\prime}\right]\right)-1}(\lambda a)=\sum_{m \geq 0} \frac{B_{m}}{m !} L_{\triangleright}\left[\Omega^{\prime}\right]^{m}(\lambda a),
$$

where the $B_{l}$ 's are the Bernoulli numbers.

Proof Let us notice that (25) can be immediately derived from (24) thanks to $L_{\triangleright}[b]=-R_{\triangleleft}[b]$ for any $b \in A$. We prove (24), which can be rewritten as

$$
\lambda a=\frac{1-\exp \left(-R_{\triangleleft}\left[\Omega^{\prime}\right]\right)}{R_{\triangleleft}\left[\Omega^{\prime}\right]}\left(\Omega^{\prime}(\lambda a)\right) .
$$

Given such $\Omega^{\prime}:=\Omega^{\prime}(\lambda a) \in \lambda \bar{A}[[\lambda]]$ we must then prove that $X:=\exp ^{*}\left(\Omega^{\prime}(\lambda a)\right)$ is the solution of $X=\mathbf{1}+\lambda a \prec X$, where $a$ is given by (26). Let us first remark that

$$
R_{\triangleleft}\left[\Omega^{\prime}\right]=R_{\prec}\left[\Omega^{\prime}\right]-L_{\succ}\left[\Omega^{\prime}\right],
$$

and that the two operators $R_{\prec}\left[\Omega^{\prime}\right]$ and $L_{\succ}\left[\Omega^{\prime}\right]$ commute thanks to the dendriform axiom (14). We have then, using the three dendriform algebra axioms,

$$
\begin{aligned}
\lambda a=\frac{1-\exp \left(-R_{\triangleleft}\left[\Omega^{\prime}\right]\right)}{R_{\triangleleft}\left[\Omega^{\prime}\right]}\left(\Omega^{\prime}\right) & =\int_{0}^{1} \exp \left(-s R_{\triangleleft}\left[\Omega^{\prime}\right]\right)\left(\Omega^{\prime}\right) d s \\
& =\int_{0}^{1} \exp \left(s L_{\succ}\left[\Omega^{\prime}\right]\right) \exp \left(-s R_{\prec}\left[\Omega^{\prime}\right]\right)\left(\Omega^{\prime}\right) d s \\
& =\int_{0}^{1} \exp ^{*}\left(s \Omega^{\prime}\right) \succ \Omega^{\prime} \prec \exp ^{*}\left(-s \Omega^{\prime}\right) d s .
\end{aligned}
$$


So we get

$$
\begin{aligned}
\lambda a \prec X & =\int_{0}^{1}\left(\exp ^{*}\left(s \Omega^{\prime}\right) \succ \Omega^{\prime} \prec \exp ^{*}\left(-s \Omega^{\prime}\right)\right) \prec \exp ^{*}\left(\Omega^{\prime}\right) d s \\
& =\int_{0}^{1} \exp ^{*}\left(s \Omega^{\prime}\right) \succ \Omega^{\prime} \prec \exp ^{*}\left((1-s) \Omega^{\prime}\right) d s \\
& =\sum_{n \geq 0} \sum_{p+q=n} \Omega^{* p} \succ \Omega^{\prime} \prec \Omega^{\prime * q} \int_{0}^{1} \frac{(1-s)^{q} s^{p}}{p ! q !} d s .
\end{aligned}
$$

An iterated integration by parts shows that

$$
\int_{0}^{1}(1-s)^{q} s^{p} d s=\frac{p ! q !}{(p+q+1) !},
$$

which yields

$$
\lambda a \prec X=\sum_{n \geq 0} \frac{1}{(n+1) !} \sum_{p+q=n} \Omega^{* * p} \succ \Omega^{\prime} \prec \Omega^{\prime * q} .
$$

On the other hand, we have

$$
X-\mathbf{1}=\exp ^{*}\left(\Omega^{\prime}\right)-\mathbf{1}=\sum_{n \geq 0} \frac{1}{(n+1) !} \Omega^{\prime * n+1} .
$$

Equality (26) then follows from the identity

$$
\sum_{p+q=n} \Omega^{* p} \succ \Omega^{\prime} \prec \Omega^{* q}=\Omega^{* n+1}
$$

which is easily shown by induction on $n$. Analogously, one readily verifies that

$$
\begin{aligned}
Y \succ \lambda a & =\int_{0}^{1} \exp ^{*}\left(-\Omega^{\prime}\right) \succ\left(\exp ^{*}\left(s \Omega^{\prime}\right) \succ \Omega^{\prime} \prec \exp ^{*}\left(-s \Omega^{\prime}\right)\right) d s \\
& =\int_{0}^{1} \exp ^{*}\left((s-1) \Omega^{\prime}\right) \succ \Omega^{\prime} \prec \exp ^{*}\left(-s \Omega^{\prime}\right) d s \\
& =\sum_{n \geq 0} \sum_{p+q=n}(-1)^{(p+q)} \Omega^{\prime * p} \succ \Omega^{\prime} \prec \Omega^{* q} \int_{0}^{1} \frac{(1-s)^{q} s^{p}}{p ! q !} d s \\
& =\sum_{n \geq 0} \frac{(-1)^{(p+q)}}{(n+1) !} \sum_{p+q=n} \Omega^{\prime * p} \succ \Omega^{\prime} \prec \Omega^{\prime * q} .
\end{aligned}
$$

Remark 3 It seems appropriate at this point to justify notation. We have chosen to write $\Omega^{\prime}$ to remind the reader of the fact that in the particular context of Example 1 (see Sect. 5.3 below) one readily sees that $\Omega^{\prime}(t)=\dot{\Omega}(t)$, see (2). 
Remark 4 In [8], we were able to show, using Hopf algebra and free Lie algebra techniques, i.e, the Dynkin idempotent map that

$$
\Omega^{\prime}(a)=\int_{0}^{1}\left(\mathcal{L}(s)+\sum_{n>0}(-1)^{n} \frac{B_{n}}{n !} a d_{\Omega^{\prime}}^{(n)}(\mathcal{L}(s))\right) d s,
$$

where $\mathcal{L}(t)=\sum_{n>0} R_{\triangleright}[a]^{(n)}(a) t^{n-1}$.

\section{The Pre-Lie Fer Expansion}

Let us come back to the dendriform power sums expansions (23):

$$
X=\mathbf{1}+\lambda a \prec X \quad \text { and } \quad Y=\mathbf{1}-\lambda Y \succ a .
$$

We will mainly focus on the first one. Following Fer's original work [9] we now make a simple ansatz for its solution:

$$
X=\exp ^{*}(\lambda a) * V_{1}
$$

and substitute this into the recursion. Recall the dendriform axiom (13). This then leads to the following:

$$
\exp ^{*}(\lambda a) * V_{1}=\mathbf{1}+\lambda a \prec\left(\exp ^{*}(\lambda a) * V_{1}\right)=\mathbf{1}+\lambda\left(a \prec \exp ^{*}(\lambda a)\right) \prec V_{1} \text {. }
$$

It is the goal to derive a recursion for $V_{1}$ similar to the original one and then to iterate the process. Remember (22) and the dendriform axiom (14):

$$
\begin{aligned}
V_{1}= & \exp ^{*}(-\lambda a)+\lambda \exp ^{*}(-\lambda a) *\left(\left(a \prec \exp ^{*}(\lambda a)\right) \prec V_{1}\right) \\
= & \exp ^{*}(-\lambda a)+\lambda \exp ^{*}(-\lambda a) \prec\left(\left(a \prec \exp ^{*}(\lambda a)\right) \prec V_{1}\right) \\
& +\lambda \exp ^{*}(-\lambda a) \succ\left(\left(a \prec \exp ^{*}(\lambda a)\right) \prec V_{1}\right) \\
= & \exp ^{*}(-\lambda a)+\lambda \exp ^{*}(-\lambda a) \prec\left(\left(a \prec \exp ^{*}(\lambda a)\right) \prec V_{1}\right) \\
& +\left(\exp ^{*}(-\lambda a) \succ \lambda a \prec \exp ^{*}(\lambda a)\right) \prec V_{1} \\
= & \mathbf{1}+\left(\exp ^{*}(-\lambda a)-\mathbf{1}\right) \prec\left(\mathbf{1}+\lambda a \prec\left(\exp ^{*}(\lambda a) * V_{1}\right)\right) \\
& +\left(\exp ^{*}(-\lambda a) \succ \lambda a \prec \exp ^{*}(\lambda a)\right) \prec V_{1} \\
= & \mathbf{1}+\left(\left(\exp ^{*}(-\lambda a)-\mathbf{1}\right) \prec \exp ^{*}(\lambda a)\right) \prec V_{1} \\
& +\left(\exp ^{*}(-\lambda a) \succ \lambda a \prec \exp ^{*}(\lambda a)\right) \prec V_{1} \\
= & \mathbf{1}+\left(\left(\exp ^{*}(-\lambda a)-\mathbf{1}\right) \prec \exp ^{*}(\lambda a)\right. \\
& \left.+\exp ^{*}(-\lambda a) \succ \lambda a \prec \exp ^{*}(\lambda a)\right) \prec V_{1} .
\end{aligned}
$$


At this point, we can repeat the above using the ansatz

$$
V_{1}:=\exp ^{*}\left(U_{1}^{\prime}\right) * V_{2}
$$

where

$$
U_{1}^{\prime}:=\left(\exp ^{*}(-\lambda a)-\mathbf{1}\right) \prec \exp ^{*}(\lambda a)+\exp ^{*}(-\lambda a) \succ \lambda a \prec \exp ^{*}(\lambda a) .
$$

In general we have

$$
V_{n}:=\exp ^{*}\left(U_{n}^{\prime}\right) * V_{n+1}
$$

with $U_{0}^{\prime}:=\lambda a$ and

$$
U_{n}^{\prime}:=\left(\exp ^{*}\left(-U_{n-1}^{\prime}\right)-\mathbf{1}\right) \prec \exp ^{*}\left(U_{n-1}^{\prime}\right)+\exp ^{*}\left(-U_{n-1}^{\prime}\right) \succ U_{n-1}^{\prime} \prec \exp ^{*}\left(U_{n-1}^{\prime}\right)
$$

Such that we arrive at the following infinite product expansion for $X$ :

$$
X=\exp ^{*}\left(U_{0}^{\prime}\right) * \exp ^{*}\left(U_{1}^{\prime}\right) * \exp ^{*}\left(U_{2}^{\prime}\right) * \cdots * \exp ^{*}\left(U_{n}^{\prime}\right) * \cdots .
$$

Analogously, using the ansatz $Y=V_{1} * \exp ^{*}(-\lambda a)$ one shows that

$$
Y=\cdots * \exp ^{*}\left(-U_{n}^{\prime}\right) * \cdots * \exp ^{*}\left(-U_{2}^{\prime}\right) * \exp ^{*}\left(-U_{1}^{\prime}\right) * \exp ^{*}\left(-U_{0}^{\prime}\right)
$$

Let us now examine a bit closer (30). By the foregoing calculation in Sect. 3 one readily verifies that

$$
\exp \left(R_{\triangleleft}[\lambda a]\right)(\lambda a)=\exp ^{*}(-\lambda a) \succ \lambda a \prec \exp ^{*}(\lambda a)
$$

Lemma 5 Let $(A, \prec, \succ)$ be a dendriform algebra augmented by a unit 1 (22). Then for $a \in A$ we have

$$
\left(\exp ^{*}(-a)-\mathbf{1}\right) \prec \exp ^{*}(a)=-\int_{0}^{1} \exp ^{*}(-s a) \succ a \prec \exp ^{*}(s a) d s .
$$

Proof In the proof of Theorem 2, we have shown (see (28), with $-a$ replacing $\Omega^{\prime}$ ) that

$$
\exp ^{*}(-a)-\mathbf{1}=-\int_{0}^{1} \exp ^{*}(-s a) \succ a \prec \exp ^{*}((s-1) a) d s .
$$

This immediately yields

$$
\begin{aligned}
& \left(\exp ^{*}(-a)-\mathbf{1}\right) \prec \exp ^{*}(a) \\
& =-\left(\int_{0}^{1} \exp ^{*}(-s a) \succ a \prec \exp ^{*}((s-1) a) d s\right) \prec \exp ^{*}(a) \\
& =-\int_{0}^{1} \exp ^{*}(-s a) \succ a \prec \exp ^{*}((s) a) d s
\end{aligned}
$$

by application of the first dendriform axiom. 
This lemma implies then for the general recursion (31)

$$
\begin{aligned}
\left(\exp ^{*}\left(-U_{n}^{\prime}\right)-\mathbf{1}\right) & \prec \exp ^{*}\left(U_{n}^{\prime}\right)+\exp ^{*}\left(-U_{n}^{\prime}\right) \succ U_{n}^{\prime} \prec \exp ^{*}\left(U_{n}^{\prime}\right) \\
& =\exp \left(R_{\triangleleft}\left[U_{n}^{\prime}\right]\right)\left(U_{n}^{\prime}\right)-\int_{0}^{1} \exp ^{*}\left(s U_{n}^{\prime}\right) \succ U_{n}^{\prime} \prec \exp ^{*}\left(-s U_{n}^{\prime}\right) d s \\
& =\exp \left(R_{\triangleleft}\left[U_{n}^{\prime}\right]\right)\left(U_{n}^{\prime}\right)-\frac{\exp \left(R_{\triangleleft}\left[U_{n}^{\prime}\right]\right)-1}{R_{\triangleleft}\left[U_{n}^{\prime}\right]}\left(U_{n}^{\prime}\right) \\
& =\exp \left(-L_{\triangleright}\left[U_{n}^{\prime}\right]\right)\left(U_{n}^{\prime}\right)+\frac{\exp \left(-L_{\triangleright}\left[U_{n}^{\prime}\right]\right)-1}{L_{\triangleright}\left[U_{n}^{\prime}\right]}\left(U_{n}^{\prime}\right) \\
& =\frac{\left(L_{\triangleright}\left[U_{n}^{\prime}\right]+1\right) \exp \left(-L_{\triangleright}\left[U_{n}^{\prime}\right]\right)-1}{L_{\triangleright}\left[U_{n}^{\prime}\right]}\left(U_{n}^{\prime}\right),
\end{aligned}
$$

and one shows that this then gives the nice identity essentially encoding Fer's classical expansion in terms of a pre-Lie product:

$$
\begin{aligned}
\left(\exp ^{*}\left(-U_{n}^{\prime}\right)-\mathbf{1}\right) & \prec \exp ^{*}\left(U_{n}^{\prime}\right)+\exp ^{*}\left(-U_{n}^{\prime}\right) \succ U_{n}^{\prime} \\
& \prec \exp ^{*}\left(U_{n}^{\prime}\right)=\sum_{l>0} \frac{(-1)^{l} l}{(l+1) !} L_{\triangleright}\left[U_{n}^{\prime}\right]^{(l)}\left(U_{n}^{\prime}\right) .
\end{aligned}
$$

In the following theorem, we summarize the above by formulating Fer's expan$\operatorname{sion}(5)$.

Theorem 6 Let $(A, \prec, \succ)$ be a dendriform algebra augmented by a unit 1 (22). Let $U_{0}^{\prime}:=\lambda a, U_{n}^{\prime}:=U_{n}^{\prime}(a), n \in \mathbb{N}, a \in A$, be elements in $\lambda \bar{A}[[\lambda]]$, such that

$$
X=\prod_{n \geq 0}^{*} \exp ^{*}\left(U_{n}^{\prime}\right), \quad Y=\prod_{n \geq 0}^{*} \exp ^{*}\left(-U_{n}^{\prime}\right)
$$

where $X$ and $Y$ are the solutions of (23). Then these elements $U_{n}^{\prime}$ obey the following recursive equation:

$$
U_{n+1}^{\prime}:=\sum_{l>0} \frac{(-1)^{l} l}{(l+1) !} L_{\triangleright}\left[U_{n}^{\prime}\right]^{(l)}\left(U_{n}^{\prime}\right), \quad n \geq 0 .
$$

The presentation given here in the context of dendriform algebras reduces to Fer's classical expansion when working in the dendriform algebra of Example 1. Let us recall that Iserles [11] rediscovered Fer's result calling it the method of iterated commutators. Munthe-Kaas and Zanna [22] further developed Iserles' work in the context of Lie group integrators.

\section{Applications}

In this section, we collect some applications where the foregoing straightforwardly implies known as well as new results. 


\subsection{Associative Algebras}

Any associative algebra $(A, *)$ can be seen as a dendriform algebra with $\prec=*$ and $\succ=0$ (or alternatively $\prec=0$ and $\succ=*$ ). In this case, the pre-Lie operation $\triangleleft$ reduces to the associative product, and (26) reduces to

$$
\lambda a=\mathbf{1}-\exp ^{*}\left(-\Omega^{\prime}\right)
$$

hence $\Omega^{\prime}=-\log ^{*}(\mathbf{1}-\lambda a)$. Its exponential $X=(\mathbf{1}-\lambda a)^{*-1}=\mathbf{1}+\lambda a+\lambda^{2} a * a+\cdots$ indeed verifies $X=\mathbf{1}+\lambda a * X$.

\subsection{Rota-Baxter Algebras}

Recall $[2,6,25]$ that an associative Rota-Baxter algebra (over a field $k$ ) is an associative $k$-algebra $A$ endowed with a $k$-linear map $R: A \rightarrow A$ subject to the following relation:

$$
R(a) R(b)=R(R(a) b+a R(b)+\theta a b),
$$

where $\theta \in k$. The map $R$ is called a Rota-Baxter operator of weight $\theta$. The map $\widetilde{R}:=-\theta i d-R$ also is a weight $\theta$ Rota-Baxter map. Both the image of $R$ and $\tilde{R}$ form subalgebras in $A$. Associative Rota-Baxter algebras arise in many mathematical contexts, e.g., in integral and finite differences calculus, but also in perturbative renormalization in quantum field theory [7].

A few examples are in order. On a suitable class of functions, we define the following Riemann summation operators

$$
R_{\theta}(f)(x):=\sum_{n=1}^{[x / \theta]} \theta f(n \theta) \text { and } R_{\theta}^{\prime}(f)(x):=\sum_{n=1}^{[x / \theta]-1} \theta f(n \theta) .
$$

Observe that

$$
\begin{aligned}
& \left(\sum_{n=1}^{[x / \theta]} \theta f(n \theta)\right)\left(\sum_{m=1}^{[x / \theta]} \theta g(m \theta)\right) \\
& \quad=\left(\sum_{n>m=1}^{[x / \theta]}+\sum_{m>n=1}^{[x / \theta]}+\sum_{m=n=1}^{[x / \theta]}\right) \theta^{2} f(n \theta) g(m \theta) \\
& =\sum_{m=1}^{[x / \theta]} \theta^{2}\left(\sum_{k=1}^{m} f(k \theta)\right) g(m \theta)+\sum_{n=1}^{[x / \theta]} \theta^{2}\left(\sum_{k=1}^{n} g(k \theta)\right) f(n \theta)-\sum_{n=1}^{[x / \theta]} \theta^{2} f(n \theta) g(n \theta) \\
& =R_{\theta}\left(R_{\theta}(f) g\right)(x)+R_{\theta}\left(f R_{\theta}(g)\right)(x)+\theta R_{\theta}(f g)(x) .
\end{aligned}
$$

Similarly for the map $R_{\theta}^{\prime}$. Hence, the Riemann summation maps $R_{\theta}$ and $R_{\theta}^{\prime}$ satisfy the weight $-\theta$ and the weight $\theta$ Rota-Baxter relation, respectively.

Let us give another example, very different from summation and integration maps. Let $A$ be a $\mathbb{K}$-algebra which decomposes directly into subalgebras $A_{1}$ and $A_{2}$, 
$A=A_{1} \oplus A_{2}$, then the projection to $A_{1}, R: A \rightarrow A, R\left(a_{1}, a_{2}\right)=a_{1}$, is an idempotent Rota-Baxter operator, i.e., of weight $\theta=-1$. Let us verify this for $a, b \in A=$ $A_{1} \oplus A_{2}$ :

$$
\begin{aligned}
R(a) b+a R(b)-a b= & R(a)(R(b)+(i d-R)(b)) \\
& -(R(a)+(i d-R)(a))(i d-R)(b) \\
= & R(a) R(b)-(i d-R)(a)(i d-R)(b)
\end{aligned}
$$

such that applying $R$ on both sides kills the term $(i d-R)(a)(i d-R)(b)$ without changing the term $R(a) R(b)$, as $R(i d-R)(a)=0$ since $A_{1}, A_{2}$ are subalgebras.

Proposition 7 [6] Any associative Rota-Baxter algebra gives a tridendriform algebra, $\left(T_{R},<,>, \bullet_{\theta}\right)$, in the sense that the Rota-Baxter structure yields three binary operations:

$$
a<b:=a R(b), \quad a>b:=R(a) b, \quad a \bullet{ }_{\theta} b:=\theta a b,
$$

satisfying the tridendriform algebra axioms (21).

The associated associative product $*_{\theta}$ is given by

$$
a *_{\theta} b:=a R(b)+R(a) b+\theta a b .
$$

It is sometimes called the "double Rota-Baxter product," and verifies

$$
R\left(a *_{\theta} b\right)=R(a) R(b), \quad \widetilde{R}\left(a *_{\theta} b\right)=-\widetilde{R}(a) \widetilde{R}(b)
$$

which is just a reformulation of the Rota-Baxter relation (35). It follows from the general link between dendriform and tridendriform algebras that any Rota-Baxter algebra gives rise to a dendriform algebra structure, $\left(D_{R}, \prec, \succ\right)$, given by

$$
a \prec b:=a R(b)+\theta a b=-a \widetilde{R}(b), \quad a \succ b:=R(a) b .
$$

For completeness, we mention the following.

Remark 8 (Rota-Baxter Dendriform algebra) It is easy to verify that $\left(D_{R}, \prec, \succ\right)$ defines a Rota-Baxter dendriform algebra with weight $\theta$ Rota-Baxter map $R$ : $D_{R} \rightarrow D_{R}$, that is

$$
\begin{aligned}
& \widetilde{R}(a) \prec \widetilde{R}(b)=\widetilde{R}(\widetilde{R}(a) \prec b+a \prec \widetilde{R}(b)+\theta a \prec b), \\
& R(a) \succ R(b)=R(R(a) \succ b+a \succ R(b)+\theta a \succ b) .
\end{aligned}
$$

This provides an example for a nonassociative Rota-Baxter algebra.

The (weight $\theta$ ) Rota-Baxter (left) pre-Lie operation corresponding to (18) is given by

$$
a \triangleright b=R(a) b-b R(a)-\theta b a=[R(a), b]-\theta b a .
$$


Let us remark that the underlying vector space $A$ equipped with associative product $*_{\theta}$ is again a Rota-Baxter algebra with weight $\theta$ Rota-Baxter map $R$. Whereas, the pre-Lie algebra $(A, \triangleright)$ is a Rota-Baxter pre-Lie algebra with weight $\theta$ RotaBaxter map $R$, i.e., another example for a nonassociative Rota-Baxter algebra. For completeness, recall the known fact that, analogously to (38), we can define a new pre-Lie product $\triangleright_{\theta}$ on $(A, \triangleright)$, and $\left(A, \triangleright_{\theta}\right)$ is again a Rota-Baxter pre-Lie algebra with weight $\theta$ Rota-Baxter map $R$.

Notice that if we suppose the algebra $A$ to be unital, the unit (which we denote by 1 ) has nothing to do with the artificially added unit $\mathbf{1}$ of the underlying dendriform algebra. We extend the Rota-Baxter algebra structure to $\bar{A}$ by setting

$$
R(\mathbf{1}):=1, \quad \widetilde{R}(\mathbf{1}):=-1 \quad \text { and } \quad \mathbf{1} \cdot x=x . \mathbf{1}=0 \quad \text { for any } x \in \bar{A} .
$$

This is consistent with the axioms (22) which in particular yield $\mathbf{1} \succ x=R(\mathbf{1}) x$ and $x \prec \mathbf{1}=-x \widetilde{R}(\mathbf{1})$, in coherence with (39).

Now let us suppose that the Rota-Baxter algebra $A$ is unital, and introduce the weight $\theta \in k$ pre-Lie Magnus type recursion, $\Omega_{\theta}^{\prime}:=\Omega_{\theta}^{\prime}(\lambda a) \in \lambda \bar{A}[[\lambda]]$, where the Rota-Baxter operator $R$ is naturally extended to $\bar{A}[[\lambda]]$ by $k[[\lambda]]$-linearity:

$$
\Omega_{\theta}^{\prime}(\lambda a)=\sum_{m \geq 0} \frac{B_{m}}{m !} L_{\triangleright}\left[\Omega_{\theta}^{\prime}\right]^{(m)}(\lambda a) .
$$

Theorem 2 together with relation (38) implies for a fixed $a \in A$ the following corollary.

Corollary 9 The elements $\hat{X}:=-\widetilde{R}(X)=\exp \left(-\widetilde{R}\left(\Omega_{\theta}^{\prime}(\lambda a)\right)\right)$ and $\hat{Y}:=R(Y)=$ $\exp \left(-R\left(\Omega_{\theta}^{\prime}(\lambda a)\right)\right)$ in $A[[\lambda]]$ solve the equations

$$
\hat{X}=1-\lambda \widetilde{R}(a \hat{X}) \quad \text { resp. } \hat{Y}=1-\lambda R(\hat{Y} a) .
$$

Proof Recall the link between dendriform and tridendriform algebras, and the RotaBaxter relation, i.e., relations (7). Using (38), we have

$$
\begin{aligned}
\hat{X} & =-\widetilde{R}(X)=-\widetilde{R}(\mathbf{1}+\lambda a \prec X)=-\widetilde{R}(\mathbf{1}-\lambda a \widetilde{R}(X)) \\
& =-\widetilde{R}(\mathbf{1}+\lambda a \hat{X})=1-\lambda \widetilde{R}(a \hat{X}),
\end{aligned}
$$

and similarly

$$
\hat{Y}=R(Y)=R(\mathbf{1}-\lambda Y \succ a)=R(\mathbf{1}-\lambda R(Y) a)=R(\mathbf{1}-\lambda \hat{Y} a)=1-\lambda R(\hat{Y} a) .
$$

We may summarize the picture we developed so far in the following diagram relating the pre-Lie Magnus expansion to the original Magnus expansion as well as to 
the Spitzer's identity:

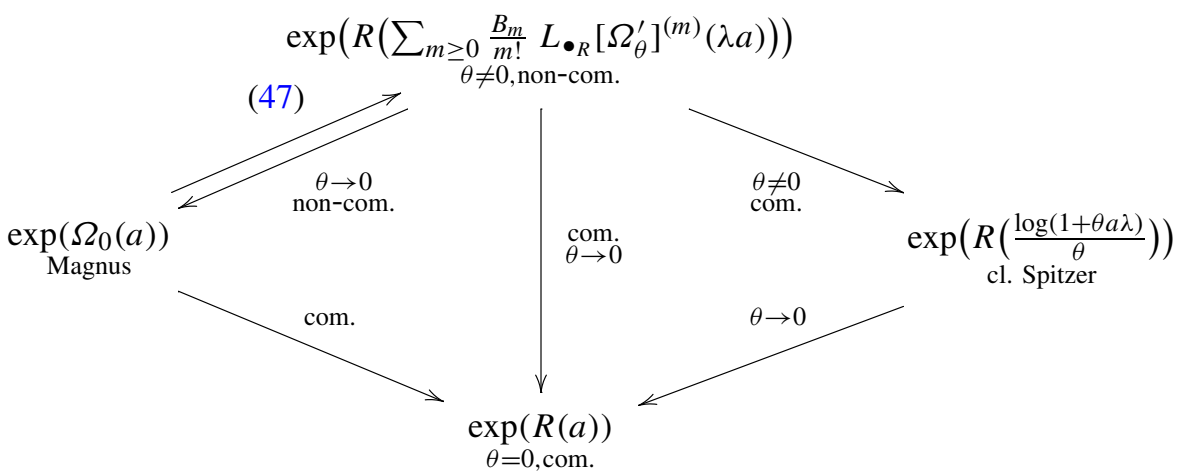

On the left side, we regard the limit $\theta \rightarrow$ zero leading to Magnus' expansion, whereas on the left side we observe the reduction of the pre-Lie Magnus expansion to the logarithm in Spitzer's identity when the underlying algebra is commutative. Finally, both formulas reduce to the classical exponential solution in a commutative weight zero Rota-Baxter algebra. We refer the reader to [7] for more details.

We define here the weight $\theta \in k$ pre-Lie Fer type recursion, $U_{n, \theta}^{\prime}:=U_{n, \theta}^{\prime}(a) \in$ $\lambda \bar{A}[[\lambda]]:$

$$
U_{n+1, \theta}^{\prime}(\lambda a)=\sum_{l>0} \frac{(-1)^{l} l}{(l+1) !} L_{\triangleright}\left[U_{n, \theta}^{\prime}\right]^{(l)}\left(U_{n, \theta}^{\prime}\right), \quad n \geq 0 .
$$

By the same line of arguments Theorem 6 implies the next corollary.

Corollary 10 The element

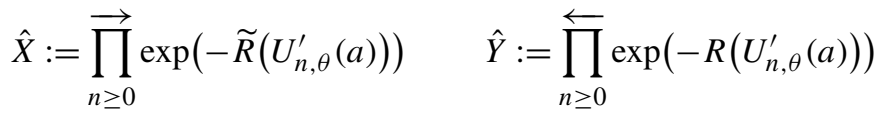

in $A[[\lambda]]$ solves the above recursions.

Associative Rota-Baxter algebras are essentially characterized by a natural factorization theorem related to the equations (43), see Atkinson [1] for more details.

Recall [7] that there is a unique (usually nonlinear) bijection $\chi_{\theta}: \lambda A[[\lambda]] \rightarrow$ $\lambda A[[\lambda]]$, which is a deformation of the identity, such that for any $\alpha \in \lambda A[[\lambda]]$ we have $^{1}$

$$
\exp (-\theta \alpha)=\exp \left(R \circ \chi_{\theta}(\alpha)\right) \exp \left(\widetilde{R} \circ \chi_{\theta}(\alpha)\right)
$$

\footnotetext{
${ }^{1}$ Notice the sign change compared to [7]. This is due to a conventional sign change in the definition of the weight: an idempotent Rota-Baxter operator is now of weight -1 with the new convention. The two recursive formulae for $\chi_{\theta}$ which follow thus differ a little bit from those given in the aforementioned reference.
} 
The so-called $\theta \mathrm{BCH}$-recursion map $\chi_{\theta}$ is recursively given by

$$
\chi_{\theta}(\alpha)=\alpha+\frac{1}{\theta} \operatorname{BCH}\left(R \circ \chi_{\theta}(\alpha), \widetilde{R} \circ \chi_{\theta}(\alpha)\right),
$$

or alternatively

$$
\chi_{\theta}(\alpha)=\alpha+\frac{1}{\theta} \operatorname{BCH}\left(\theta \alpha, R \circ \chi_{\theta}(\alpha)\right),
$$

where $\operatorname{BCH}(x, y)$ is the Baker-Campbell-Hausdorff series defined by $\exp (x) \exp (y)$ $=\exp (x+y+\operatorname{BCH}(x, y))$. Recall Atkinson's theorem [1]:

$$
\hat{Y}(1-\theta \lambda a) \hat{X}=1,
$$

hence $\hat{Y}^{-1} \hat{X}^{-1}=1-\theta \lambda a$. We deduce immediately from the very definitions of $\hat{X}$ and $\hat{Y}$ that we have

$$
1-\theta \lambda a=\exp \left(-\theta \alpha_{\theta}\right)=\exp \left(R\left(\Omega_{\theta}^{\prime}\right)\right) \exp \left(\widetilde{R}\left(\Omega_{\theta}^{\prime}\right)\right)
$$

with $\alpha_{\theta}:=\alpha_{\theta}(\lambda a):=-\frac{1}{\theta} \log (1-\theta \lambda a)$. We then infer from (45) the equality

$$
\Omega_{\theta}^{\prime}=\Omega_{\theta}^{\prime}(\lambda a)=\chi_{\theta}\left(\alpha_{\theta}\right)=\chi_{\theta}\left(-\frac{\log (1-\theta \lambda a)}{\theta}\right),
$$

which was conjectured in [4] in a similar context. From (46), we obtain, for any $\alpha \in \lambda A[[\lambda]]$,

$$
\chi_{\theta}(\alpha)=\Omega_{\theta}^{\prime}\left(\frac{1-\exp (\theta \alpha)}{\theta}\right)
$$

\subsection{The Weight Zero Case and the Classical Magnus Expansion}

For a weight $\theta=0$, Rota-Baxter algebra the pre-Lie product (40) reduces to

$$
a \triangleright b=[R(a), b] .
$$

This simplifies the weighted pre-Lie Magnus type recursion (42) to the classical Magnus recursion

$$
\Omega_{0}^{\prime}(\lambda a)=\chi_{0}(\lambda a)=\sum_{m \geq 0} \frac{B_{m}}{m !} L_{\triangleright}\left[\chi_{0}\right]^{(m)}(\lambda a)=\sum_{m \geq 0} \frac{B_{m}}{m !} a d_{R\left(\chi_{0}\right)}^{(m)}(\lambda a) .
$$

An important example for a weight zero Rota-Baxter algebra is any algebra $F$ of operator-valued functions on the real line, closed under integrals $\int_{0}^{x}$, say, smooth $n \times n$ matrix-valued functions. Recall example (1), showing that $F$ is a dendriform algebra. The associative product then writes

$$
A * B(x):=A(x) \cdot \int_{0}^{x} B(y) d y+\int_{0}^{x} A(y) d y \cdot B(x)
$$


such that

$$
\exp \left(\int_{0}^{x} A(y) d y\right)=\int_{0}^{x}\left(\exp ^{*}(A(y))\right) d y .
$$

Recall our convention (41) for the dendriform unit. Then the classical Magnus recursion (2), $\Omega(t)=\Omega(A)(t)$, for $R:=\int_{0}^{t}$ reads

$$
\begin{aligned}
\dot{\Omega}(A)(t) & =\Omega_{0}^{\prime}(A)(t)=\sum_{m \geq 0} \frac{B_{m}}{m !} L_{\triangleright}[\dot{\Omega}(A)]^{(m)}(A)(t) \\
& =\sum_{m \geq 0} \frac{B_{m}}{m !} a d_{\int_{0}^{t} \dot{\Omega}(A)(s) d s}^{(m)}(A(t)) .
\end{aligned}
$$

Similarly, in the case of the weighted pre-Lie Fer expansion, as expected, we recover the original formula.

We showed in reference [7] that the recursion $\chi_{\theta}$ reduces to the classical Magnus recursion (49) in the limit $\theta \rightarrow 0$ :

$$
\chi_{\theta}(\lambda a) \stackrel{\theta \rightarrow 0}{\longrightarrow} \chi_{0}(\lambda a)
$$

We recover this result from (47), (42), and from the fact that $\alpha_{\theta}(\lambda a) \rightarrow \lambda a$ when $\theta \rightarrow 0$. Hence, we have proven that the weight $\theta \mathrm{BCH}$-recursion $\chi_{\theta}$ derives naturally from the classical Magnus recursion via the nonlinear change of variable $\alpha_{\theta}$.

Let us finish this brief note with an interesting observation to be further explored in a future work. In the context of Example 1 and the initial value problem (1), we have seen that the classical Magnus and Fer recursion can be rewritten using the (weight zero) pre-Lie product introduced by the integral operator $\int_{0}^{x}$ :

$$
\dot{\Omega}_{0}(A)(s)=\sum_{m \geq 0} \frac{B_{m}}{m !} L_{\triangleright}\left[\dot{\Omega}_{0}(A)(s)\right]^{(m)}(A(s)),
$$

and

$$
\dot{U}_{n+1,0}(A)(s)=\sum_{l>0} \frac{(-1)^{l} l}{(l+1) !} L_{\triangleright}\left[\dot{U}_{n, 0}(A)(s)\right]^{(l)}\left(\dot{U}_{n, 0}(A(s))\right) .
$$

It seems to be natural to ask whether the extra pre-Lie structure in the case of the Magnus as well as the Fer expansion implies a possible reduction in the number of terms. Here we would like to indicate that this is the case, by explicit verification up to fifth order in the case of the Magnus expansion, using the dendriform algebra, respectively, the induced pre-Lie product presented in Example 1. We should emphasize the fact, that the following applies, of course, to the more general weight $\theta$ pre-Lie Magnus and Fer type recursions (42), (44), respectively.

We introduce a dummy parameter $\lambda$ for convenience. Obviously, as the (left) preLie relation (18) is a ternary one, we expect it to be available only from third order upwards in this parameter: 


$$
\begin{aligned}
\Omega(t)= & \int_{0}^{t} \dot{\Omega}_{0}(A \lambda)(s) d s \\
= & \lambda \int_{0}^{t} A(s) d s-\lambda^{2} \frac{1}{2} \int_{0}^{t} A \triangleright A(s) d s \\
& +\lambda^{3} \int_{0}^{t}\left(\frac{1}{12}(A \triangleright(A \triangleright A))(s)+\frac{1}{4}((A \triangleright A) \triangleright A)(s)\right) d s \\
& +\lambda^{4} \int_{0}^{t}\left(-\frac{1}{8}((A \triangleright A) \triangleright A) \triangleright A(s)-\frac{1}{24}(A \triangleright(A \triangleright A)) \triangleright A(s)\right. \\
& \left.-\frac{1}{24}(A \triangleright((A \triangleright A) \triangleright A)(s)+(A \triangleright A) \triangleright(A \triangleright A)(s))\right) d s+\mathcal{O}(5) .
\end{aligned}
$$

Recall that $A \triangleright A(s)=\left[\int_{0}^{s} A(u) d u, A(s)\right]$. We see that at third order no further reduction of terms is possible. At fourth order, we find a reduction to two terms using the pre-Lie relation (18). Indeed, one verifies that

$$
\begin{aligned}
& \frac{1}{8}((A \triangleright A) \triangleright A) \triangleright A \\
& \quad+\frac{1}{24}((A \triangleright(A \triangleright A)) \triangleright A+A \triangleright((A \triangleright A) \triangleright A)+(A \triangleright A) \triangleright(A \triangleright A))
\end{aligned}
$$

using that thanks to the pre-Lie relation

$$
(A \triangleright A) \triangleright(A \triangleright A)=((A \triangleright A) \triangleright A) \triangleright A-(A \triangleright(A \triangleright A)) \triangleright A+A \triangleright((A \triangleright A) \triangleright A)
$$

equals

$$
\frac{1}{6}((A \triangleright A) \triangleright A) \triangleright A+\frac{1}{12} A \triangleright((A \triangleright A) \triangleright A) .
$$

At fifth order we observe a reduction in the number of terms from ten to seven. More details and a complete analysis of this apparently new structure in the Magnus (and Fer) expansion will be provided in a forthcoming work.

Following the seminal work of Iserles and Nørsett [12, 13], using planar rooted binary trees to encode the combinatorial setting in Magnus expansion, we may present the foregoing calculation more transparently. The binary tree

$$
\Lambda \sim A \triangleright A .
$$

At fourth order, we have

$$
\frac{1}{8} \widehat{\Lambda}+\frac{1}{24}(\widehat{\lambda}+\lambda+\lambda \lambda)
$$

which reduces to

$$
\frac{1}{6} \widehat{\Lambda}+\frac{1}{12} \lambda
$$


thanks to the left pre-Lie relation

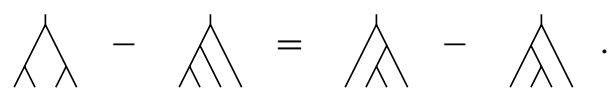

Acknowledgements The first named author acknowledges greatly the support by the European PostDoctoral Institute. He thanks A. Iserles, H. Munthe-Kaas and B. Owren for helpful discussions, and the Department of Applied Mathematics and Theoretical Physics at Cambridge University for warm hospitality. A special thanks goes to A. Völzmann-Scheiding at MPIM Bonn. We would like to thank Frédéric Fauvet for inviting us to Institut de Recherche Mathématique Avancée, Université Louis Pasteur where part of this work was done. We would like to thank Frédéric Patras and Jose M. Gracia-Bondía for helpful discussions, and Jean-Louis Loday for useful comments.

\section{References}

1. F.V. Atkinson, Some aspects of Baxter's functional equation, J. Math. Anal. Appl. 7, 1-30 (1963).

2. G. Baxter, An analytic problem whose solution follows from a simple algebraic identity, Pac. J. Math. 10, 731-742 (1960).

3. S. Blanes, F. Casas, J.A. Oteo, J. Ros, Magnus and Fer expansions for matrix differential equations: the convergence problem, J. Phys. A: Math. Gen. 31, 259-268 (1998).

4. J. Cariñena, K. Ebrahimi-Fard, H. Figueroa, J.M. Gracia-Bondía, Hopf algebras in dynamical systems theory, Int. J. Geom. Method. Mod. Phys. 4(4), 577-646 (2007).

5. F. Chapoton, Un théorème de Cartier-Milnor-Moore-Quillen pour les algèbres dendriformes et les algèbres braces, J. Pure Appl. Algebra 168, 1-18 (2002).

6. K. Ebrahimi-Fard, Loday-type algebras and the Rota-Baxter relation, Lett. Math. Phys. 61, 139-147 (2002).

7. K. Ebrahimi-Fard, L. Guo, D. Manchon, Birkhoff type decompositions and the Baker-CampbellHausdorff recursion, Commun. Math. Phys. 267, 821-845 (2006).

8. K. Ebrahimi-Fard, D. Manchon, F. Patras, New identities in dendriform algebras, J. Algebra (2008 to appear). arXiv:0705.2636v1 [math.CO].

9. F. Fer, Résolution de l'equation matricielle $\dot{U}=p U$ par produit infini d' exponentielles matricielles, Bull. Classe Sci. Acad. R. Belg. 44, 818-829 (1958).

10. I.M. Gelfand, D. Krob, A. Lascoux, B. Leclerc, V. Retakh, J.-Y. Thibon, Noncommutative symmetric functions, Adv. Math. 112, 218-348 (1995). arXiv:hep-th/9407124.

11. A. Iserles, Solving linear differential equations by exponentials of iterated commutators, Numer. Math. 45, 183-199 (1984).

12. A. Iserles, Expansions that grow on trees, Not. AMS 49, 430-440 (2002).

13. A. Iserles, S.P. Nørsett, in Geometric Integration: Numerical Solution of Differential Equations on Manifolds, ed. by C.J. Budd, A. Iserles. Philosophical Transactions of the Royal Society A, vol. 357 (London Mathematical Society, London, 1999), pp. 983-1020.

14. A. Iserles, H.Z. Munthe-Kaas, S.P. Nørsett, A. Zanna, Lie-group methods, Acta Numer. 9, 215-365 (2000).

15. M. Kawski, Chronological algebras: combinatorics and control, Itogi Nauk. Tech. 68, 144-178 (2000).

16. S. Klarsfeld, J.A. Oteo, Recursive generation of higher-order terms in Magnus expansion, Phys. Rev. A 39, 3270-3273 (1989).

17. J.-L. Loday, Cup-product for Leibniz cohomology and dual Leibniz algebras, Math. Scand. 77(2), 189-196 (1995).

18. J.-L. Loday, in Dialgebras. Lect. Notes Math., vol. 1763 (Springer, Berlin, 2001), pp. 7-66.

19. J.-L. Loday, M. Ronco, Homotopy theory: relations with algebraic geometry, group cohomology, and algebraic K-theory, Contemp. Math. 346, 369-398 (2004).

20. W. Magnus, On the exponential solution of differential equations for a linear operator, Commun. Pure Appl. Math. 7, 649-673 (1954).

21. B. Mielnik, J. Plebański, Combinatorial approach to Baker-Campbell-Hausdorff exponents, Ann. Inst. Henri Poincaré A XII, 215-254 (1970). 
22. H. Munthe-Kaas, A. Zanna, in Foundations of Computational Mathematics, ed. by F. Cucker, M. Shub (Springer, Berlin, 1997), pp. 434-441.

23. J.A. Oteo, J. Ros, From time-ordered products to Magnus expansion, J. Math. Phys. 41, 3268-3277 (2000).

24. M. Ronco, Primitive elements in a free dendriform algebra, Contemp. Math. 207, 245-263 (2000).

25. G.-C. Rota, Baxter algebras and combinatorial identities. I, II., Bull. Am. Math. Soc. 75, 325-329 (1969); ibidem 330-334.

26. M.P. Schützenberger, in Séminaire Dubreil-Jacotin Pisot (Algèbre et théorie des nombres), Paris, Année 1958/59.

27. R.S. Strichartz, The Campbell-Baker-Hausdorff-Dynkin formula and solutions of differential equations, J. Funct. Anal. 72, 320-345 (1987).

28. R.M. Wilcox, Exponential operators and parameter differentiation in quantum physics, J. Math. Phys. 8, 962-982 (1967).

29. A. Zanna, Technical Report 1996/NA12, Department of Applied Mathematics and Theoretical Physics, University of Cambridge. 